VOL. 60 (1999) [191-196]

\title{
A BOUND ON THE SCHUR MULTIPLIER OF A PRIME-POWER GROUP
}

\author{
Graham Ellis and James Wiegold
}

\section{For Bernhard Neumann on his 90th birthday}

The paper improves on an upper bound for the order of the Schur multiplier of a finite $p$-group given by Wiegold in 1969. The new bound is applied to the problem of classifying $p$-groups according to the size of their Schur multipliers.

In a paper [6] dedicated to B.H. Neumann's sixtieth birthday, the second author used results of [5] to show that a $d$-generator group $G$ of prime-power order $p^{n}$ has Schur multiplier $M(G)$ of order at most $p^{(d-1)(2 n-d) / 2}$. In this article we use results of the first author [3] to obtain a reduction of this bound. The reduced bound is then applied to the problem of classifying $p$-groups according to the orders of their Schur multipliers, at least in the case where the multipliers are large.

We begin by blending parts (i) and (ii) of Proposition 5 in [3] to produce the following proposition.

Proposition 1. [3] Let $G$ be a finite p-group with centre $Z(G)$ and lower central series $1=\gamma_{c+1} G \unlhd \gamma_{c} G \unlhd \cdots \unlhd \gamma_{1} G=G$. Set $\bar{G}=G / Z(G)$ and consider the homomorphism

$\Psi: \bar{G}^{a b} \otimes \bar{G}^{a b} \otimes \bar{G}^{a b} \longrightarrow \frac{\gamma_{2} G}{\gamma_{3} G} \otimes \bar{G}^{a b}, \bar{x} \otimes \bar{y} \otimes \bar{z} \mapsto[x, y]_{\gamma} \otimes \bar{z}+[y, z]_{\gamma} \otimes \bar{x}+[z, x]_{\gamma} \otimes \bar{y}$

Here $\bar{x}$ denotes the image in $\bar{G}$ of the element $x \in G$, and $[x, y]_{\gamma}$ denotes the image in $\gamma_{2} G / \gamma_{3} G$ of the commutator $[x, y] \in G$. Then

$$
|M(G)|\left|\gamma_{2} G\right||\operatorname{image}(\Psi)| \leqslant\left|M\left(G^{a b}\right)\right|\left|\frac{\gamma_{2} G}{\gamma_{3} G} \otimes \bar{G}^{a b}\right|\left|\frac{\gamma_{3} G}{\gamma_{4} G} \otimes \bar{G}^{a b}\right| \cdots\left|\gamma_{c} G \otimes \bar{G}^{a b}\right|
$$

Proposition 1 leads to the following numerical bound on the order of the Schur multiplier.

Received 19th April, 1999

The first author was based at the Max-Planck-Institut für Mathematik, Bonn during the course of this work. He would like to thank the Institute for its very generous hospitality.

Copyright Clearance Centre, Inc. Serial-fee code: 0004-9727/99 \$A2.00+0.00. 
TheOREM 2. Let $G$ be a $d$-generator group of order $p^{n}$. Suppose that the Abelianisation $G^{a b}$ has order $p^{m}$ and exponent $p^{e}$, and that the central quotient $G / Z(G)$ is a $\delta$-generator group. Then

$$
|M(G)| \leqslant p^{d(m-e) / 2+(\delta-1)(n-m)-\max \{0, \delta-2\}}
$$

Since $e \geqslant m / d$ and $d \geqslant \delta$, inequality (2) implies

$$
|M(G)| \leqslant p^{(d-1)(2 n-m) / 2}
$$

Bound (3) is attained if $G=C_{p^{e}} \times C_{p^{e}} \times \cdots \times C_{p^{e}}$.

Proof: Recall that $M\left(G^{a b}\right)$ is isomorphic to the exterior square $G^{a b} \wedge G^{a b}$ of Abelian groups [2]. Suppose that $G^{a b} \cong C_{p^{n_{1}}} \times C_{p^{n_{2}}} \times \cdots \times C_{p^{n_{d}}}$ where $n_{1} \leqslant n_{2} \leqslant$ $\cdots \leqslant n_{d}=e$ and $n_{1}+n_{2}+\cdots+n_{d}=\mathrm{m}$. Then $M(G)$ has order $p^{a}$, where

$$
\begin{aligned}
a & =(d-1) n_{1}+(d-2) n_{2}+\cdots+n_{d-1} \\
& =d\left(n_{1}+n_{2}+\cdots+n_{d-1}\right)-\left(n_{1}+2 n_{2}+\cdots+(d-1) n_{d-1}\right) \\
& =d(m-e)-\left(n_{1}+2 n_{2}+\cdots+(d-1) n_{d-1}\right) \\
& \leqslant d(m-e)-\frac{m-e}{d-1}(1+2+\cdots+(d-1)) \\
& =d(m-e) / 2 .
\end{aligned}
$$

Since the tensor product is distributive with respect to direct sums, we have

(5) $\left|\frac{\gamma_{2} G}{\gamma_{3} G} \otimes \bar{G}^{a b}\right|\left|\frac{\gamma_{3} G}{\gamma_{4} G} \otimes \bar{G}^{a b}\right| \cdots\left|\gamma_{c} G \otimes \bar{G}^{a b}\right|=\left|\left(\frac{\gamma_{2} G}{\gamma_{3} G} \oplus \cdots \oplus \gamma_{c} G\right) \otimes \bar{G}^{a b}\right| \leqslant p^{\delta(n-m)}$

Suppose next that $\delta \geqslant 3$. Since $\gamma_{2} G / \gamma_{3} G$ is non-trivial, we can choose a generating set $\left\{\bar{x}_{1}, \bar{x}_{2}, \cdots, \bar{x}_{\delta}\right\}$ for $G / Z(G)$ such that $\left[x_{1}, x_{2}\right]_{\gamma}$ is a non-trivial element of $\gamma_{2} G / \gamma_{3} G$ and indeed is not a $p$ th power of any element there since $p$ th powers lie in the Frattini subgroup. We shall establish now the critical point of the proof, namely that the $\delta-2$ elements

$$
\Psi\left(\bar{x}_{1} \otimes \bar{x}_{2} \otimes \bar{x}_{3}\right), \Psi\left(\bar{x}_{1} \otimes \bar{x}_{2} \otimes \bar{x}_{4}\right), \cdots, \Psi\left(\bar{x}_{1} \otimes \bar{x}_{2} \otimes \bar{x}_{\delta}\right)
$$

constitute $\delta-2$ linearly independent elements in the Abelian group $\gamma_{2} G / \gamma_{3} G \otimes \bar{G}^{a b}$. Setting $A:=\gamma_{2} G / \gamma_{3} G$ temporarily, we see that

$$
A \otimes \bar{G}^{a b} \cong\left(A \otimes\left\langle\bar{x}_{1}\right\rangle\right) \times \cdots \times\left(A \otimes\left\langle\bar{x}_{\delta}\right\rangle\right)
$$


and that $\Psi\left(\bar{x}_{1} \otimes \bar{x}_{2} \otimes \bar{x}_{i}\right)$ is the only one of the $\delta-2$ elements listed in (6) above to have a non-trivial projection in $A \otimes\left\langle\bar{x}_{i}\right\rangle$, so that these $\delta-2$ elements are indeed linearly independent and we have

$$
|\operatorname{image}(\Psi)| \geqslant p^{\delta-2}
$$

Inequality (2) is obtained by substituting inequalities (4), (5) and (7) into (1).

The methods in $[3]$ show that the quantity $|\operatorname{image}(\Psi)|$ could be replaced by a (larger) product $|\operatorname{image}(\Psi)|\left|\operatorname{image}\left(\Psi_{3}\right)\right| \cdots\left|\operatorname{image}\left(\Psi_{c}\right)\right|$ in inequality (1), thus leading to an improvement in the bounds of Theorem 2.

On substituting the inequalities $d \leqslant m \leqslant n$ into (3) we obtain a well-known result of J.A. Green, namely that $|M(G)| \leqslant p^{n(n-1) / 2}$ for any group $G$ of order $p^{n}$. In other words, for any group $G$ of order $p^{n}$ there is an integer $t \geqslant 0$ such that $|M(G)|=p^{n(n-1) / 2-t}$. Those finite $p$-groups with $t=0,1$ have been classified by Berkovich [1]. The classification has been extended to $t=2$ by Zhou [7], and to $t=3$ by the first author [4]. In light of this work we make the following formal definition.

Definition: Let the corank of a finite $p$-group $G$ be the integer $t=\operatorname{corank}(G)$ for which $|M(G)|=p^{n(n-1) / 2-t}$ with $n=\log _{p}|G|$.

The known classifications of finite $p$-groups by corank are summarised in the following table. All groups $G$ with corank $(G) \leqslant 3$ are listed. In the table $C_{p^{i}}$ denotes the cyclic group of order $p^{i}, D$ denotes the dihedral group of order $8, Q$ denotes the quaternion group of order $8, E_{1}$ denotes the extraspecial group of order $p^{3}$ with odd exponent $p$, and $E_{2}$ denotes the extraspecial group of order $p^{3}$ with odd exponent $p^{2}$.

$$
\begin{array}{lll}
\operatorname{corank}(G) & p=2 & p=\text { odd prime } \\
t=0 & \left(C_{2}\right)^{k}, k \geqslant 1 & \left(C_{p}\right)^{k}, k \geqslant 1 \\
t=1 & C_{4} & C_{p^{2}}, E_{1} \\
t=2 & C_{2} \times C_{4}, D & C_{p} \times C_{p^{2}}, E_{1} \\
t=3 & C_{8}, C_{2} \times C_{2} \times C_{4}, Q, D \times C_{2} & C_{p^{3}}, C_{p} \times C_{p} \times C_{p^{2}}, E_{2}, E_{1} \times C_{p} \times C_{p}
\end{array}
$$

In [4] it is shown how the information in this table can be derived from a bound on the Schur multiplier due to Gaschütz, Neubüser and Yen [5]. Since inequality (2) is slightly sharper than the bound of Gaschütz et alia, it too has ramifications for the classification of $p$-groups by corank. Some of these are listed in the following proposition. An interesting corollary to the proposition is that, for any given prime $p$ and integer $t \geqslant 1$, there are only finitely many $p$-groups $G$ with $\operatorname{corank}(G)=t$.

PROPOSITION 3. Let $G$ be a non-cyclic $d$-generator group of order $p^{n}$, with commutator subgroup $[G, G]$ of order $p^{c}$, and Frattini subgroup $[G, G] G^{p}$ of order $p^{a}$. 
Suppose that the Abelianisation $G^{a b}$ has exponent $p^{e}$, and that the central quotient $G / Z(G)$ is a $\delta$-generator group. Furthermore, suppose that corank $(G)=t$ where $t \geqslant 1$. Then:

(i) $0 \leqslant c \leqslant t$.

(ii) $c \leqslant a \leqslant \sqrt{2 t-c}$.

(iii) $2 \leqslant d \leqslant\left(2(t+a)-a^{2}-3 c\right) /(a-c)$ whenever $a \neq c$.

(iv) $2 \leqslant d \leqslant t+2-\left(a^{2}+a\right) / 2$ whenever $a=c$.

(v) $\quad\left(a^{2}-2 a+(d+3) c+a d-2(t+1)\right) /(2 c-1) \leqslant \delta \leqslant d$ whenever $c \neq 0$.

(vi) $1 \leqslant e \leqslant\left(2 t-2(d+1-\delta) c-d(a-c-1)-\left(a^{2}-a\right)-2 \max \{0, \delta-2\}\right) / d$.

(vii) $(1+\sqrt{1+4 t}) / 2 \leqslant n \leqslant(2 t+a(c+e)+2(\delta-1) c-$ $2 \max \{0, \delta-2\}) /(c+e+a-1)$.

Proof: Note that $a \geqslant c \geqslant 0, d \geqslant \delta \geqslant 0, e \geqslant m / d \geqslant 1$ and $d \geqslant 2$. On substituting $n=a+d, m=a+d-c$ into (2) we obtain

$$
a^{2}-a \leqslant 2(t-(d+1-\delta) c)+d(c+1-a-e)-2 \max \{0, \delta-2\} .
$$

We derive the inequality

$$
a^{2}-a \leqslant 2(t-c)-(a-c)(d-1)-2 \max \{0, \delta-2\}
$$

from (8) by substituting $d \geqslant \delta, e \geqslant m / d$. Since $a^{2}-a \geqslant 0$, inequality (9) implies (i). Since $d-1 \geqslant 1$, inequality (9) implies $a^{2} \leqslant 2 t-c$, from which we deduce (ii). We also deduce (iii) from (9). On substituting $a=c, e=1, \delta \geqslant 2$ into (8), we obtain

$$
d+(d-\delta)(a-1) \leqslant t+2-\frac{a^{2}+a}{2} .
$$

The inequality $\delta \geqslant 2$ corresponds to the fact [2] that no non-trivial cyclic group is itself a central quotient. Inequality (10) implies (iv). Inequality (9) implies (v), the condition $c \neq 0$ being used to obtain $\delta \geqslant 2$. Inequality (8) implies (vi) and the righthand inequality of (vii). The left-hand inequality of (vii) follows immediately from the definition of corank.

Corollary 4.

(i) For each prime $p$ and integer $t \geqslant 0$ there exists at least one $p$-group with corank equal to $t$.

(ii) For each prime $p$ and integer $t \geqslant 1$ there are only finitely many $p$-groups with corank equal to $t$.

Proof: The formula for the Schur multiplier of a direct product [2], namely $M(G \times H) \cong M(G) \oplus M(H) \oplus\left(G^{a b} \otimes H^{a b}\right)$, can be used to show that the Abelian 
group $\left(C_{p}\right)^{t-1} \times C_{p^{2}}$ has corank equal to $t$ for each $t \geqslant 1$. Any elementary Abelian group has corank equal to 0 . This proves part (i).

Suppose that $G$ is a p-group with $\operatorname{corank}(G)=t \geqslant 1$. Proposition 3 implies that the order of $G$ is bounded by a number, say $f(t)$, that depends only on $t$. There are only finitely many groups of order at most $f(t)$. This proves part (ii).

The following modification to the definition of corank provides a single numerical parameter for measuring how far a $p$-group 'deviates' from being elementary Abelian.

Definition: The relative corank of a finite $p$-group $G$ is the number

$$
\operatorname{rcrank}(G)=\frac{\operatorname{corank}(G)}{\log _{p}|G|}
$$

Thus the relative corank is a rational number lying in the range

$$
0 \leqslant \operatorname{rcrank}(G) \leqslant \frac{\log _{p}|G|-1}{2} .
$$

Proposition 3(ii) shows that groups with a small relative corank also have a relatively small Frattini subgroup. But relative corank captures more than the size of the Frattini subgroup. For example, the dihedral and quaternion groups of order eight have $\operatorname{rcrank}(D)=2 / 3$ and $\operatorname{rcrank}(Q)=1$. For certain families of groups it is fairly straightforward to compute the relative corank. For instance, letting $E S(p, k)$ denote an arbitrary extraspecial $p$-group of order $p^{2 k+1}$, we have:

$$
\begin{aligned}
& \operatorname{rcrank}\left(\left(C_{p}\right)^{n}\right)=0, \\
& \operatorname{rcrank}\left(C_{p^{n}}\right)=(n-1) / 2, \\
& \operatorname{rcrank}\left(\left(C_{p}\right)^{n-2} \times C_{p^{2}}\right)=(n-1) / n, \\
& \operatorname{rcrank}\left(\left(C_{p^{2}}\right)^{n / 2}\right)=n / 4, \\
& \operatorname{rcrank}(E S(p, k))=1, \text { for } k \geqslant 2, \\
& \operatorname{rcrank}(E S(p, k) \times E S(p, k))=2+1 /(4 k+2), \text { for } k \geqslant 2 .
\end{aligned}
$$

To obtain the last two calculations we have used the description of the Schur multipliers of extraspecial $p$-groups given in [2], together with the following simple lemma whose proof is left to the reader.

Lemma 5. Let $G$ and $H$ be groups of orders $p^{n}$ and $p^{m}$. Then

$$
\operatorname{rcrank}(G \times H)=\frac{n}{n+m} \operatorname{rcrank}(G)+\frac{m}{n+m} \operatorname{rcrank}(H)+\frac{n m-\log _{p}\left|G^{a b} \times H^{a b}\right|}{n+m} .
$$




\section{REFERENCES}

[1] Ya.G. Berkovich, 'On the order of the commutator subgroup and Schur multiplier of a finite p-group', J. Algebra 144 (1991), 269-272.

[2] F.R. Beyl and J. Tappe, Group extensions, representations, and the Schur multiplicator, Lecture Notes in Math. 958 (Springer-Verlag, Berlin, Heidelberg, New York, 1982).

[3] G. Ellis, 'A bound for the derived and Frattini subgroups of a prime-power group', Proc. Amer. Math. Soc. 126 (1998), 2513-2523.

[4] G. Ellis, 'On the Schur multiplier of $p$-groups', Comm. Algebra (to appear).

[5] W. Gaschütz, J. Neubüser and Ti Yen, 'Über den Multiplikator von $p$-Gruppen', Math. Z. 100 (1967), 93-96.

[6] J. Wiegold, 'Commutator subgroups of finite p-groups', J. Austral. Math. Soc. 10 (1969), 480-484.

[7] X. Zhou, 'On the order of Schur multipliers of finite p-groups', Comm. Algebra 22 (1994), $1-8$.

Max-Planck-Institut für Mathematik

Gottfried-Claren-Strasse 26

D-53225 Bonn

Germany

and

Department of Mathematics

National University of Ireland

Galway

Ireland

e-mail: graham.ellis@nuigalway.ucg.ie
School of Mathematics

Cardiff University

Senghenydd Road

Cardiff CF2 4YH

Wales 